\title{
Comment
}

Cell Death and Differentiation (2004) 11, 1364. doi:10.1038/sj.cdd.4401514

In their reply to my Letter to the Editor, O'Reilly and coworkers have now concluded that FADD is in both the nucleus and cytoplasm rather than in the cytoplasm alone. This is diplomatic - two previous publications showed FADD to be mainly nuclear - however, still of questionable veracity. First, O'Reilly et al. argue that saponin-permeabilized cells permit efficient nuclear staining, using p53 as an example. As I stated/showed in my letter, some (probably not all) antibodies for nuclear proteins fail to detect a nuclear signal in saponinpermeabilized cells. Antibodies differ, and the counterexample, p53, does not prove that the saponin method is valid for FADD antibodies, which the data clearly suggest that it is not. Secondly, they claim that their nuclear fractionation method would have retained FADD in the nuclear fraction if it was there, because PARP was retained efficiently. However, PARP is tightly chromatin-associated and a much larger protein than FADD, thus, not an appropriate control for leakage of nuclear proteins during fractionation - a prohibitive problem for this method in some cases. Finally, they attempted to resolve the issue by immunogold-EM localization of FADD. The images shown in their letter reveal roughly equal numbers of gold particles in the nucleus and cytoplasm. However, the numbers here are extremely small so it is unclear what the ratio of particles will be once quantitation is performed.

\section{S Frisch}

Mary Babb Randolph Cancer Center, POB 9300, West Virginia University, Morgantown, WV 26506-9300, USA

* Corresponding author: S Frisch; E-mail: sfrisch@hsc.wvu.edu 